\title{
Notch Filtering of humming GSM mobile telephone noise
}

\author{
Ingvar Claesson \\ Blekinge Institute of Technology \\ SE 37225 Ronneby, Sweden
}

\author{
Andreas Rossholm \\ Ericsson $\mathrm{AB}$ \\ SE 22100 Lund, Sweden
}

\begin{abstract}
A common problem in the world's most widespread cellular telephone system, the GSM system, is the interfering signal generated in TDMA cellular telephony. The infamous "bumblebee" is generated by the switching nature of TDMA cellular telephony, the radio circuits are switched on and off at a rate of approximately $217 \mathrm{~Hz}$ (GSM).

This paper describes a study of two solutions for eliminating the humming noise with IIR notch filters. The simpler one is suitable for any exterior equipment. This method still suffers from a small residual of the noise, resulting from the IDLE slots of the sending mobile. The more advanced IIR structure for use within the mobile also eliminates this residual.
\end{abstract}

\section{INTRODUCTION}

In GSM mobile telephony it is a common problem that an interfering signal is introduced into the microphone signal when the mobile is transmitting. This interfering signal is transmitted along with the speech signal to the receiver. Due to the humming sound of the interfering signal it is commonly denoted the Bumblebee.

Since interleaving of data is utilized and since control data transmission is also necessary, the connection between transmitter/receiver frames and speech frames is somewhat complicated. The interference consists of the fundamental frequency and its harmonics, where the fundamental switching rate is approximately $217 \mathrm{~Hz}$, more specifically, 5200/(3 . 8) $\mathrm{Hz}$, according to the GSM standard [1]. Signals are sent in chunks of data, speech frames, equivalent to 160 samples of data corresponding to $20 \mathrm{~ms}$ at $8 \mathrm{kHz}$ sampling rate. Data from a speech frame of $20 \mathrm{~ms}$ is sent in several bursts, each occupying $1 / 8$ of a transmitting frame. The radio circuits are switched on and off with the radio access rate frequency. An electromagnetic field pulsating with this frequency and its harmonics disturbs its own microphone signal, as well as electronic equipment in the vicinity (within 1-2 meters) of the sending handset antenna, such as radios and active loudspeakers as well as hearing aids, producing in some cases annoying periodic humming noise in the uplink speech from the handset to the base station.

It has been proposed that for internal cancelation in the mobile, the periodic distorsion can be removed by subtraction of an estimate of the distorsion employing correlators and subtraction, similar to Active Noise Control [2], [3], [4]. This estimate can be done, since it is known at what frequencies the disturbance will occur, by correlating the block of data with a number of base functions. These base functions are blocks of data corresponding to the fundamental tone and its harmonics. The results of the correlations are used to estimate the amplitude and phase of the bumblebee.

However for equipment with no access to the internal data sending structure of the GSM mobile, notch filters is still the most straight-forward solution.

\section{BACKGROUND AND ANALYSIS OF THE BUMBLEBEE}

A typical recorded disturbed signal from a silent room can be seen in Figure 1. The interfering signal is periodic but somewhat complicated since, in the case of Full Rate transmission, FR, there is no transmission when the mobile is listening to other base stations. Such silent frames occur once every 26 TDMA-frames and are denoted idle frames, see Figure 2.

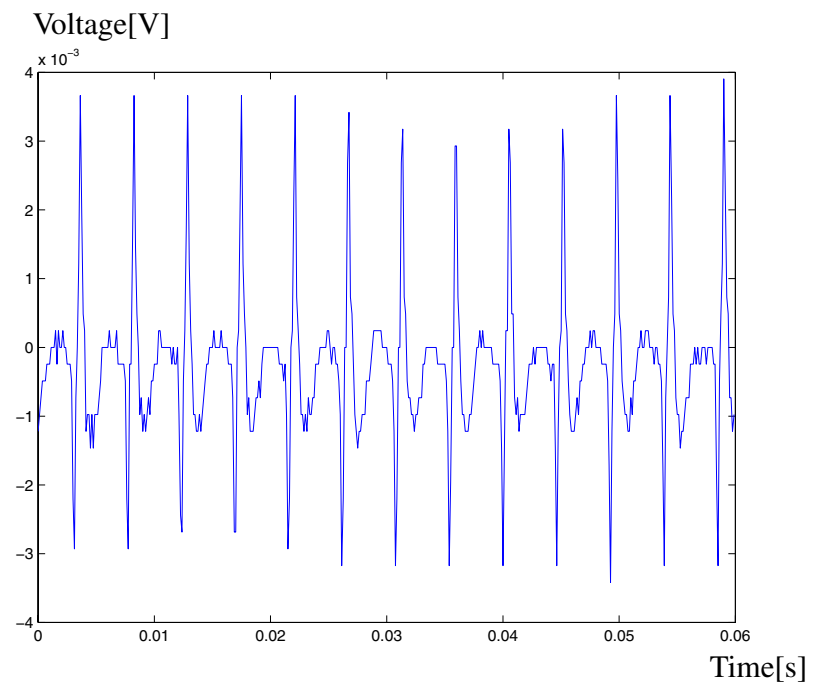

Fig. 1. Interfering signal at the microphone A/D converter recorded in a silent room with no speech.

In densely populated areas, such as Hong Kong, an alternative is sometimes used, Half Rate Transmission (HR), offering cheaper traffic with slightly decreased speech quality. In this case, the period of the interference is $1 /(8 \cdot 2 \cdot(3 / 5200)) \approx 108$ $\mathrm{Hz}$, which is half the frequency of the FR, since the mobile is only transmitting during every other time slot, thus enabling almost twice the number of calls as compared to Full Rate Transmission.

In the HR case the disturbance pattern is thus even more complex,see Fig 3, but observe that since the state of the 


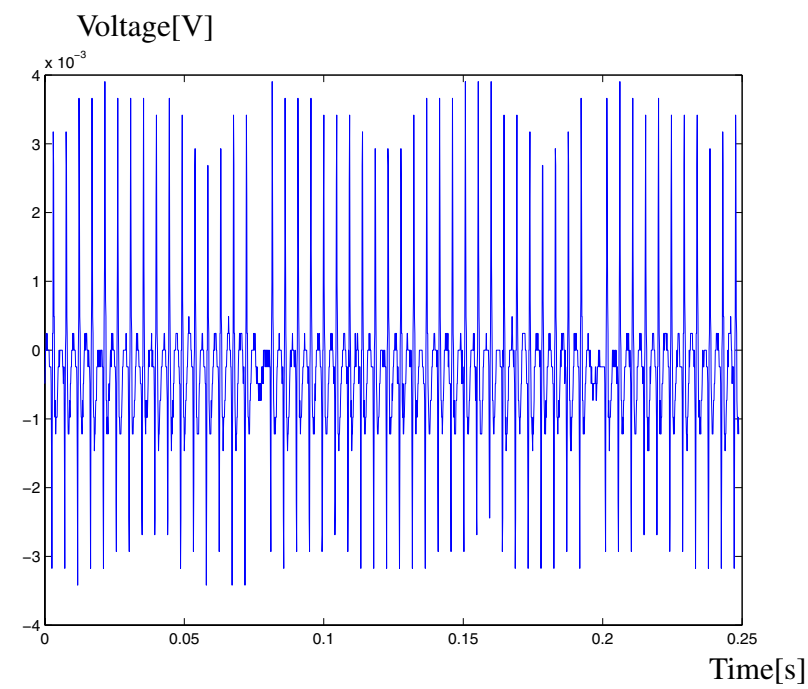

Fig. 2. Pattern for interfering signal recorded in a silent room, Full Rate.

communication between the mobile and base station is known, sufficient information to perform internal cancellation is always at hand.

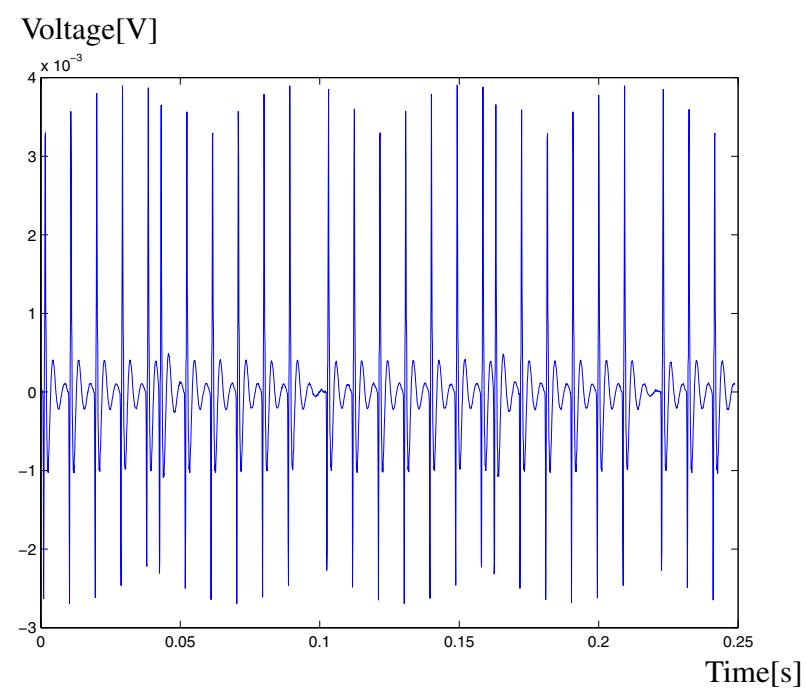

Fig. 3. Pattern for interfering signal recorded in a silent room, Half Rate.

Suppressing the "bumblebee" noise by analog means is a costly, time-consuming and difficult work. It may also require non-optimal system settings in, e.g., the microphone gain, as well as more expensive components. If a digital method is employed, it must be able to continuously track variations in the amplitude and phase of the disturbing periodic signal. The reason for this is that the conditions may change during a call, e.g., the amplitudes are a function of the output power level, and the phases a function of the timing towards the air IF (time slot). Since these parameters change during a call, we must be able to cope with this. Making a Fourier series expansion of the disturbing periodic signal, it is seen that the frequency components decay as $1 / \mathrm{f} 2$, which is very slow. In other words there are approximately 15 frequency components that must be suppressed in the band below $3.4 \mathrm{kHz}$.

By using base function correlation [4] we must save blocks of data in memory, which is negligible with notch filters. Also, this estimation can only be done during speech pauses, which makes it dependent on side information like Voice Activity Detection(VAD).

A notch filter contains deep notches, in its frequency response. Such a filter is useful when specific frequency components of known frequencies must be eliminated [5], [6]. To eliminate the frequencies at $\omega_{n}, n=[1, \ldots, N]$, pairs of complex-conjugated nulls and zeros are placed on and just inside the unit circle at the angles $\omega_{n}$

$$
z_{n_{1,2}}=r_{b} e^{ \pm j \omega_{n}}, \quad r_{b}=1
$$

Consequently, the system function of the resulting notch filter is

$$
H(z)=\frac{B(z)}{A(z)}=b_{o} \prod_{n=1}^{N} \frac{\left(1-r_{b} e^{j \omega_{n}} z^{-1}\right)\left(1-r_{b} e^{-j \omega_{n}} z^{-1}\right)}{\left(1-r_{a} e^{j \omega_{n}} z^{-1}\right)\left(1-r_{a} e^{-j \omega_{n}} z^{-1}\right)}
$$

where

$$
b_{0}=\frac{\sum_{n=1}^{N} a_{n}}{\sum_{n=1}^{N} b_{n}}
$$

Using a single, simple straight-forward notch filter will reduce the disturbance significantly, but not totally. This problem is related to the radio access pattern in GSM. In GSM, the mobile makes one radio access every $4.615 \mathrm{~ms}$. Unfortunately, the mobile does not transmit during every time slot. In one $120 \mathrm{~ms}$ multiframe, there are 26 TDMA frames of $4.615 \mathrm{~ms}$ each, i.e., there are 26 possible occasions for the mobile to transmit. However, only 24 of them are required for transmission of speech coded data (frames 0-11 and 13-24), and one for transmission of the SAACH control data (frame 12). The problem is TDMA frame 25 , the idle frame, in which there is no radio transmission. During the idle frame (or idle time slot), the mobile measures neighboring cells. Since the radio of the mobile is not transmitting during the idle frame, the disturbance is zero during this period, and the IIR filters are trying to cancel a noise that is not there.

A simple Notch filter is an IIR (infinite-duration impulse response) filter, attenuating the "bumblebee", but introduces a new residual disturbance. The frequency of the introduced disturbance is approximately $8 \mathrm{~Hz}(=1 / 120 \mathrm{~ms})$. Although the disturbing power is much attenuated compared to the original "bumblebee" signal, the fluttering characteristic of the introduced noise is still perceived. Because of the absence of radio transmission in the idle frame the "bumblebee" noise is not exactly periodic with the TDMA frame rate, even though it appears so when listening to it.

\section{Notch SOLuTIONS}

\section{A. Simple Notch filter}

We first apply a notch filter directly in the signal path to reduce the interference. The notches are made as deep as 
possible, so that ideally the frequencies in question are totally eliminated. This results in the following system function:

$$
\frac{B(z)}{A(z)}=\frac{\sum_{k=1}^{16} a_{k}}{\sum_{k=1}^{16} b_{k}} \prod_{k=1}^{16} \frac{\left(1-r_{b} e^{j k \omega_{0}} z^{-1}\right)\left(1-r_{b} e^{-j k \omega_{0}} z^{-1}\right)}{\left(1-r_{a} e^{j k \omega_{0}} z^{-1}\right)\left(1-r_{a} e^{-j k \omega_{0}} z^{-1}\right)}
$$

The calculations are made recursively on the whole data set. This will result in a convergence period at the start up and also when a handover between base stations occurs. Unfortunately, the notch filter is active also under idle frames, a drawback resulting from the fact that it works sample-bysample and recursively, leading to small, residual artifacts during idle frames, when trying to subtract a disturbance that is not present, i.e. a negative disturbance is added, see Figures 4-6.

Power Spectrum $[\mathrm{dB}]$

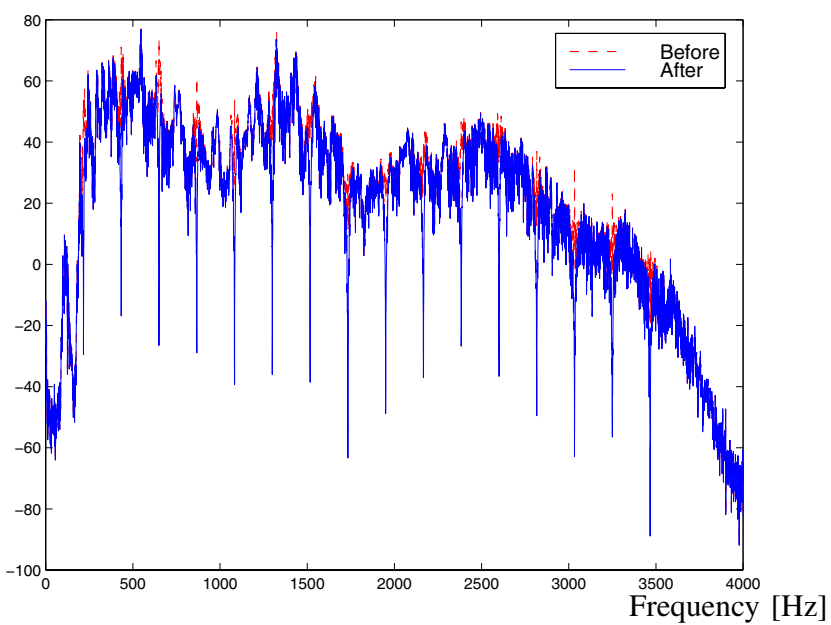

Fig. 4. Cancelation of the Bumblebee with notch filter in speech . Full Rate. Voltage [V]

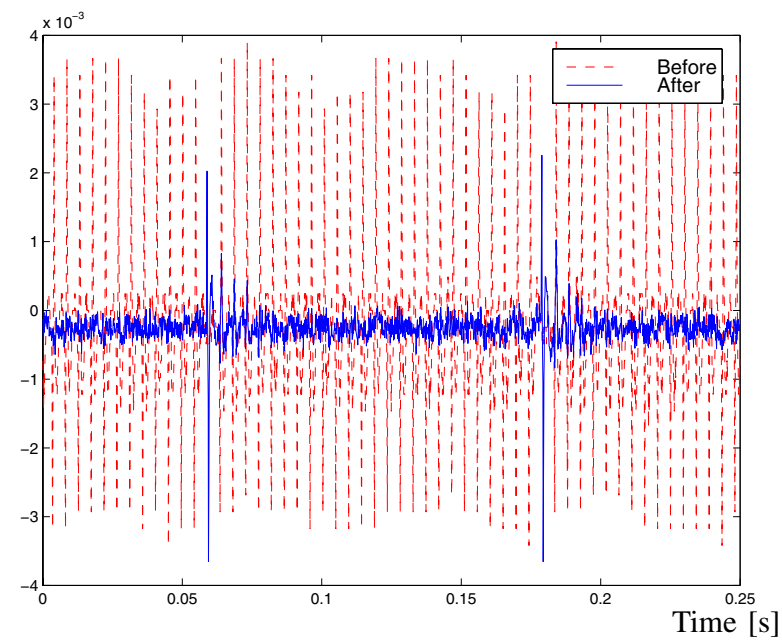

Fig. 5. Time signal of the simple notched Bumblebee. Observe residual in blue in idle slots, which is eliminated by advanced solution.
Power Spectrum $[\mathrm{dB}]$

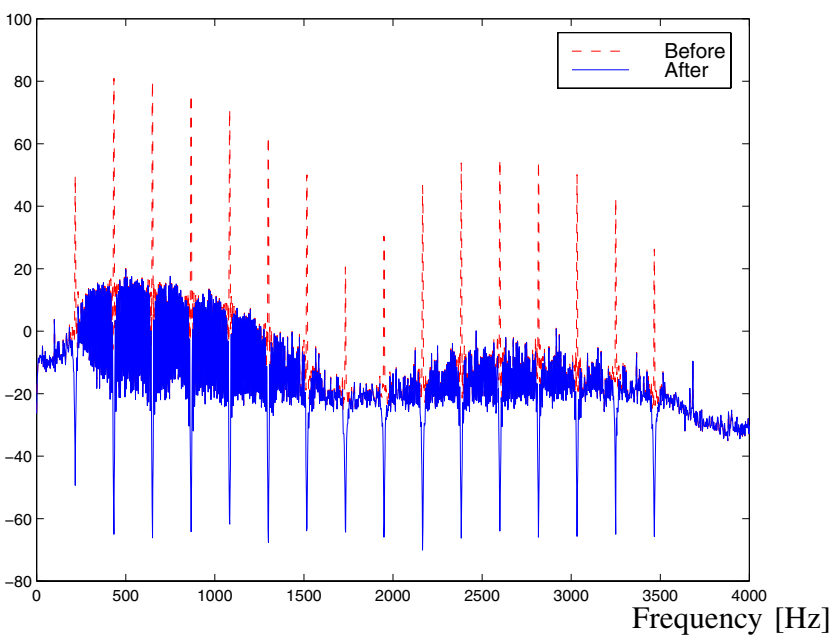

Fig. 6. Cancelation of the Bumblebee with notch filter. The Bumblebee was recorded in a silent room. Full Rate, no speech.

It can however be seen that the Bumblebee disturbance is considerably attenuated. However, this solution can be further improved to even more satisfactory results, by handling the residual periodic interference, 26 times lower in frequency, see Figure 5. The reason for this is that the notch filter consists of poles (autoregressive), which give feedback of the output signal $(y(t))$ continuously. Consequently, the Bumblebee is added during the idle frame, according to the tails of impulse responses of IIR filters.

\section{B. Advanced Notch filter}

We propose the following solution to the problem for internal cancelation the mobile. We make use of our a priori knowledge that the disturbing signal consists of a sum of sinusoids of very well known frequencies, i.e., the disturbing signal can be expressed as

$$
e(k)=\sum_{1}^{N} A_{n} \sin \left(2 \pi k n f_{0} / f s+\varphi_{n}\right)
$$

where $f_{0}=216.66 \ldots H z(=3 \cdot 8 / 5200 \mathrm{~ms})$, is the fundamental frequency, and $f_{s}=8 \mathrm{kHz}$, the sampling frequency in GSM), $1 \leq n \leq 15$, and finally $A_{n}$ and $\varphi_{n}$ are the amplitude and phase of frequency component $\mathrm{n}$, respectively.

We again make use of our knowledge about the location of the idle frame in the PCM sample stream. This can be done since communication to the DSP during a call is performed with code and decode commands from the host ASIC. A code command requires a reply from the DSP containing speech coded data from the 160 latest received PCM samples. The code commands arrive to the DSP on the average every $20 \mathrm{~ms}$. However, their exact time arrivals follow the pattern (18.465 ms, $18.465 \mathrm{~ms}, 23.070 \mathrm{~ms}$ ), i.e., over a period of three code commands the average distance is $20 \mathrm{~ms}$.

In order for the DSP to be able to synchronize its PCM buffers properly, the code commands contain information on 


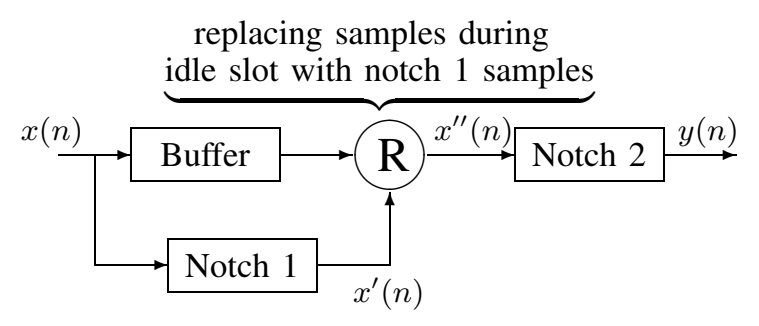

Fig. 7. Two-stage notch filtering

the time to the next code command, the "syncInfo". This information can take six different values, and is carried in the code commands in a three bit field. When synchronizing the PCM buffers, we only make use of the two least significant bits in the field, since they contain sufficient information for that task.

The interesting fact about the "syncInfo" information is that each of the six possible numbers corresponds to a certain position in the $120 \mathrm{~ms}$ multi-frame structure. (Each multiframe of $120 \mathrm{~ms}$ corresponds to six code commands to the DSP.) Thus, given the "syncInfo" information in the code commands, it is possible to calculate the position of the idle burst!

The basic idea is to now use two notch filters, that notch the bumblebee, and the "syncInfo". The difference between the notch filters is that one of the two filters has slightly larger notch-bandwidth. The first filter is only used to insert the distorsion during the idle slot, which was the problem with a single notch filter. These samples are then used to replace the samples in the original signal during the idle slot. The idle slot is located by using "syncInfo". This results in that the bumblebee signal gets present during the idle slot and from that it follows that the signal is periodic with the TDMA frame rate. The second filter is then used to notch the new signal with the periodic bumblebee. The reason for the difference in bandwidth is to make sure that we do not adding any distorsion that is not suppressed.

The first filter, denoted "Notch 1", has the smallest bandwidth. It is used to notch the input signal $x(n)$, During the idle slot, the samples in $\mathrm{x}(\mathrm{n})$ are replaced by samples from the notched signal $x^{\prime}(n)$, containing the residual bumblebee ringing out from the states in the IIR filters. By changing these samples, the new signal (x"(n)) includes a complete bumblebee, even during the idle slot. The second filter, "Notch 2 ", then notches this signal, which suppresses the bumblebee without any residual disturbance and negligible distorsion.

\section{SUMmARY AND CONCLUSIONS}

This paper presents two notch filter based solutions to reduce the humming disturbance in GSM mobile telephony. The first is a straight-forward solution with notch filters, reducing the disturbance considerably, but not totally. The second solution is a dual cascaded notch filter solution with internal knowledge of the GSM transmission pattern and transmitter state.With this method a full elimination of the "Bumblebee" can be achieved.

While the simple method is appropriate for exterior electronic equipment, the second more advanced cancelation is suited for internal cancelation in the mobile telephone.

\section{REFERENCES}

[1] GSM Standard (GSM 05.01 version 7.0.0 Release 1998) Digital cellular telecommunications system (Phase 2+), Physical layer on the radio path (General description).

[2] B.Widrow, S. D. Stearns Adaptive Signal Processing Prentice Hall, 1985

[3] M. Kuo, D. R. Morgan, Active Noise Control Systems, John Wiley \& Sons, Inc., 1996.

[4] I. Claesson, A. Nilsson GSM TDMA Frame Rate Internal Active Noise Cancellation. International Journal of Acoustics and Vibration (IJAV), vol. 8, no. 3, 2003.

[5] Proakis, J.G. and Manolakis, D.G. Digital signal processing, pp. 343345, 1996, Prentice-Hall Inc.

[6] Simon Haykin Digital communications, 1988, John Wiley \& Sons Inc.

[7] Peyton Z. Peebles, Jr. Probability, random variables, and random signal principles, 1993, McGraw-Hill Inc.

[8] Steven M. Kay Fundamentals of statistical signal processing: Estimation Theory, pp. 183-198, 1993, Prentice-Hall Inc.

[9] Per Eriksson On estimation of the amplitude and the phase function (Technical Report TR-148), 1981, University of Lund / SWEDEN. 